\title{
Avaliação de estratégia para identificação e mensuração dos acidentes de trabalho fatais
}

\author{
Evaluation of the strategy for identification \\ and measurement of the fatal accidents at work
}

Eliane de Freitas Drumond ${ }^{1}$

Jussara de Medeiros Silva ${ }^{2}$

\footnotetext{
${ }^{1}$ Nível Central, Gerência de Epidemiologia e Informação, Secretaria Municipal de Saúde de Belo Horizonte. Av. Afonso Pena 2336/9o, Funcionários. 30130007 Belo Horizonte MG. eliane_drumond@ yahoo.com.br ${ }^{2}$ Gerência de Saúde do Trabalhador, Secretaria Municipal de Saúde de Belo Horizonte.
}

\begin{abstract}
In order to evaluate the strategy used by the Municipal Health Department of Belo Horizonte (SMSA/BH) for reducing the under-reporting of deaths from work-related accidents during the years of 2008 to 2010, a deterministic comparison of data on deaths from work-related accidents as reported in SINAN and SIM was conducted. As a complementary strategy to the deterministic comparison, the Investigation Data Sheets and Death Certificates were analyzed, which although not clearly declared, were suspected of involving death by work-related accident. Death from work accident was confirmed when the same victim, same accident, with a temporal connection between accident and death, were matched. The complementary strategy used by SMSA reduced the under-reporting identified by deterministic linking, with an increase of 45 deaths. Higher rates of under-reporting of deaths in the SINAN $(n=117)$ were found than in the SIM $(n=70)$. Although data linkage between SIM/SINAN is a necessary strategy to reduce the under-reporting of deaths from accidents, it is still insufficient, considering the limitations still present in both systems. The complementary strategy adopted by SMSA/BH, which is simple and easy to perform, yields good results.
\end{abstract}

Key words Occupational accident reporting, Information systems, Occupational mortality, Under-reporting, Death
Resumo Objetivando avaliar a estratégia da Secretaria Municipal de Saúde de Belo Horizonte (SMSA/BH) na redução do sub-registro de mortes por acidentes de trabalho de 2008 a 2010, realizou-se relacionamento determinístico de dados de mortes por acidentes de trabalho notificadas no SINAN e no SIM. Como estratégia complementar ao relacionamento determinístico foram analisadas as Fichas de Investigação e as Declarações de Óbito nas quais se suspeitava tratar-se de mortes por acidentes de trabalho, porém elas não estavam declaradas. O óbito por acidente de trabalho era confirmado quando se tratava do mesmo acidentado, mesmo acidente, com nexo temporal entre o acidente e o óbito. A estratégia complementar utilizada na SMSA permitiu reduzir a sub-registro identificado pelo relacionamento determinístico, com incremento de 45 mortes. Evidenciou-se maior sub-registro de óbitos no SINAN $(n=117)$ do que no SIM $(n=70)$. Concluise que o relacionamento de dados SIM/SINAN é uma estratégia necessária para redução da subregistro das mortes por acidentes de trabalho. Porém, consideradas as limitações ainda presentes nos dois sistemas o relacionamento de dados não é suficiente e que a estratégia complementar adotada na SMSA/BH - simples e de fácil execução, tem bons resultados.

Palavras-chave Notificação de acidentes de trabalho, Sistemas de Informação, Mortalidade ocupacional, Sub-registro, Morte 


\section{Introdução}

O elevado percentual de sub-registros de mortes por acidentes de trabalho é bastante conhecido no Brasil ${ }^{1}$. A maioria dos estudos brasileiros sobre o tema basearam-se em dados obtidos junto ao Ministério da Previdência Social. Também no Sistema de Informações sobre Mortalidade (SIM), sob gestão do Ministério da Saúde e alimentado pelas declarações de óbito, observa-se significativa subestimação das mortes por acidentes de trabalho no Brasil ${ }^{2,3}$. A magnitude do problema é ainda maior para os trabalhadores não cobertos pela seguridade social, entre os quais são observados elevados e ainda mais invisíveis coeficientes de mortalidade por acidentes de trabalho ${ }^{2}$.

O Ministério da Saúde, por meio da Portaria 777 de 28 de abril de $2004^{4}$, estabeleceu a notificação compulsória de 11 agravos relacionados ao trabalho. Esta, buscava atender à necessidade de informações consistentes e ágeis sobre a situação da produção, do perfil dos trabalhadores e da ocorrência de agravos relacionados ao trabalho. Buscava também orientar as ações de saúde e considerando a constatação de que essas informações estão dispersas, fragmentadas e pouco acessíveis no âmbito do SUS. Para tal, implantou a Ficha de Investigação (FI) de acidente de trabalho grave, que alimenta o Sistema de Informação de Agravos de Notificação (SINAN). Por meio desse sistema deve ser feita notificação de todos os acidentes de trabalho graves, independentemente da vinculação trabalhista do acidentado, tenham eles ocorrido no exercício da atividade laboral ou no percurso de casa para o trabalho e vice versa. São considerados acidentes de trabalho graves aqueles que resultam em morte, em mutilações e os que acontecem em menores de dezoito anos. Em Belo Horizonte (BH), as Comunicações de Acidentes de Trabalho (CAT) são também utilizadas como fontes para preenchimento das Fichas de Investigação que alimentam o SINAN, implantado desde 2007 no município.

$\mathrm{O}$ adequado dimensionamento dos níveis e tendências reais das mortes por acidentes de trabalho no Brasil relaciona-se, principalmente, a dois fatores - sub-registro e subinformação. $\mathrm{O}$ sub-registro de informações resulta do não preenchimento da FI, da Declaração de Óbito (DO) ou de ambas. A subinformação resulta do preenchimento incorreto das FI e DO, quando se omite que o acidente de trabalho resultou em morte e/ou que a morte relacionou-se ao trabalho. Tal omissão ocorre, entre outros fatores, devido ao desconhecimento dos profissionais da saúde sobre o correto preenchimento e à importância desses documentos.

O presente estudo tem como objetivo avaliar estratégia utilizada na Secretaria Municipal de Saúde e Assistência de Belo Horizonte (SMSA$\mathrm{PBH}$ ) na identificação de mortes por acidentes de trabalho.

\section{Métodos}

Estudo descritivo baseado no relacionamento determinístico dos óbitos ocorridos em $\mathrm{BH}$ e dos de residentes no município ocorridos em outras localidades por AT, obtidos no SINAN 5 e no SIM ${ }^{6}$ em 2008 a 2010. De forma complementar, foram selecionadas FI e DO que continham informações parciais sugestivas de óbitos por acidente de trabalho, como segue. No SINAN foram avaliadas a ocupação do acidentado, a gravidade, a data, o local e a circunstância do acidente; no SIM a ocupação do acidentado, data do óbito, local e circunstância do acidente. A estratégia utilizada na identificação dos óbitos por AT foi composta pelas seguintes etapas:

1. No SINAN, a partir do campo 66 - Evolução do Caso (5- óbito por acidente de trabalho grave). Para suprir as deficiências na notificação de acidente de trabalho grave conforme preconizado pelo Ministério da Saúde, a alimentação do SINAN em BH é feita principalmente a partir das informações constantes nas Comunicações de Acidente de Trabalho, obtidas por busca ativa em unidades sentinela selecionadas do município, referências para atendimento de urgência e emergência. Cópias das FI assim selecionadas foram encaminhadas à Gerência de Epidemiologia e Informação para cruzamento dessas informações com as do SIM.

2. No SIM, a partir do campo 57 - Acidente de Trabalho (1-Sim). Em BH, os óbitos por causas externas (códigos do Capítulo XX da CID 10) são investigados rotineiramente. As circunstâncias dessas mortes são obtidas no Instituto Médico Legal de BH (IML), a partir de informações de boletins de ocorrência, hospitais, famílias e outras fontes. As informações dos óbitos por acidentes de trabalho também são obtidas no IML. Cópias das DO cuja causa da morte era relacionada ao trabalho foram encaminhadas à Gerência de Saúde do Trabalhador para cruzamento dessas informações com as do SINAN.

3. Relacionamento determinístico das informações e sua incorporação a cada um dos Sistemas. 
4. Cruzamento e a análise das informações parciais contidas no SINAN e no SIM. Nessa etapa, foram cruzadas as informações das FI, cujo campo 66 - evolução do caso, o código 5 (óbito por acidente de trabalho grave) não estava assinalado com as informações das DO nas quais o campo 57- acidente de trabalho, o código 1 ( $\operatorname{sim}$ ) não estava preenchido. Foram utilizadas as seguintes variáveis para certificar de que se tratava da mesma ocorrência: nome, ocupação e data de nascimento do falecido; nome da mãe, municípios de ocorrência e de residência, data, local e circunstância do acidente. Quando verificado que se tratava do mesmo acidentado e do mesmo acidente (avaliado por meio do intervalo de tempo decorrido entre o AT e o óbito), assinalavamse os respectivos campos. Ligações telefônicas e visitas às empresas onde ocorreram os acidentes e notícias (jornais e televisão) auxiliaram na confirmação dos casos. Quando confirmados como óbitos por acidente de trabalho, os registros eram incluídos no SINAN e no SIM.

5. Avaliação do sub-registro nos dois sistemas e em cada um.

\section{Resultados}

Segundo dados da SMSA (atualizados em 31/05/ 2012), no período analisado havia no SIM 4.421 óbitos por causas externas na população de 16 a 65 anos residente em $\mathrm{BH}$, dos quais 99 devidos a acidentes de trabalho. Já no SINAN estavam registrados 4.962 acidentes de trabalho grave, com 88 óbitos. Apenas 29 dos óbitos por AT foram notificados simultaneamente nos dois sistemas de informação.
A etapa de relacionamento determinístico possibilitou a identificação de 194 mortes por acidentes de trabalho. Houve incorporação de 106 notificações do SINAN, o que significou redução de sub-registro de 54,6\% (106/194) e de 48,9\% (95/194) no SIM. Na etapa complementar, de cruzamento e análise das informações parciais provenientes dos dois sistemas, identificaram-se mais 45 óbitos. Desta maneira, ao final do processo, obtiveram-se 239 óbitos por acidente de trabalho.

Em relação ao perfil dos acidentados, 94,3\% eram homens, negros (47,5\%), com menos de 8 anos de estudo (36,9\%). Apenas um trabalhador era menor de 18 anos, mas sete deles tinham mais de 65 anos. A maioria (86,5\%) residia em BH ou na sua região metropolitana. Observou-se elevado percentual de não informação $(43,3 \%)$ no que se refere à situação no mercado de trabalho. Quanto à ocupação, houve maior freqüência de acidentes entre trabalhadores da indústria extrativa e da construção civil (25,5\%), seguidos dos trabalhadores dos serviços diversos (motociclistas e vendedores do comércio - 18,4\%) e trabalhadores de funções transversais (condutores de veículos - 18,4\%). A via pública foi o local de ocorrência da maioria das mortes $(50,4 \%)$. Tratava-se de acidente típico em 59,6\%, de trajeto em 22,0\%, mas em 18,4\% essa informação não estava disponível. Em 74,5\% dos casos $(n=105)$ o óbito ocorreu no mesmo dia do acidente e em $14,9 \%(n=21)$ em até cinco dias após. A importância dos acidentes de transporte e das quedas pode ser vista na Tabela 1 .

Ressalte-se a ocorrência de 31 homicídios, em sua maioria provocados por agressões por arma de fogo que não puderam ser notificados no SIM,

Tabela 1. Distribuição das circunstâncias de óbitos por acidentes de trabalho em Belo Horizonte, 2008-2010.

\begin{tabular}{lrr}
\hline \multicolumn{1}{c}{ CID Acidente } & Frequência & Percentual \\
\hline Acidentes de transporte & 101 & $42,3 \%$ \\
Quedas & 42 & $17,6 \%$ \\
Exposição a forças mecânicas inanimadas (Ex: impacto causado por objetos, & 41 & $17,2 \%$ \\
explosões, projéteis de armas de fogo, contato com facas, dentre outros) & 31 & $13,0 \%$ \\
Agressões & 10 & $4,2 \%$ \\
Exposição a corrente elétrica & 5 & $2,0 \%$ \\
Exposição à fumaça, ao fogo e às chamas & 5 & $2,0 \%$ \\
Risco a respiração devido a desmoronamento, queda de terra e de outras substâncias & 3 & $1,3 \%$ \\
Exposição a forças mecânicas animadas & 1 & $0,4 \%$ \\
Intervenção legal & 239 & $100,0 \%$ \\
Total & & \\
\hline
\end{tabular}

Fonte: SINAN NET 
devido ao filtro do sistema - homicídios não podem ser classificados como acidentes de trabalho. Em apenas 33,3\% dos casos, os homicídios enquadravam-se como acidentes de trajeto. Quanto aos típicos, destacam-se entre as principais ocupações deste grupo os condutores de veículos, porteiros e frentistas.

\section{Discussão}

A estratégia utilizada para a redução do sub-registro de óbitos por acidente de trabalho apresentada nesse estudo revelou-se adequada. Ela soma-se a outras propostas ${ }^{7-9}$ com vistas a suprir as falhas de informações sobre óbitos por acidentes de trabalho e obter sua real dimensão por meio de estatísticas oficiais.

A precariedade das informações pode ser observada tanto nas FI quanto nas DO. Contribuem para essa situação as dificuldades relatadas pelos médicos para o preenchimento da DO, a inexistência de fluxo claro e objetivo para a notificação dos acidentes de trabalho graves, a falta de conhecimento e sensibilização dos profissionais de saúde para a necessidade e a obrigatoriedade de notificação dos acidentes de trabalho graves e fatais. Deve ser citado, também, o receio de posterior implicação jurídica e/ou retaliação pelos agentes envolvidos contra o profissional acidentado e/ ou o profissional de saúde notificador ${ }^{10}$.

Apesar de sua reconhecida limitação ${ }^{11}$ a utilização das CAT, obtidas em Belo Horizonte por meio de busca ativa em unidades sentinelas reduziu, ainda que parcialmente, o sub-registro de acidentes de trabalho grave no SINAN. Ainda é necessário o desenvolvimento de ações visando conscientizar profissionais de saúde, trabalhadores e toda a sociedade sobre a importância do cumprimento da obrigação legal de notificação compulsória dos acidentes de trabalho graves e fatais. Para superar a deficiência de qualidade das informações, além do cruzamento das mesmas, foi necessário o trabalho integrado dos pesquisadores com experiência na área para a seleção dos casos considerados suspeitos. A partir dessa suspeição realizava-se o cruzamento e a busca ativa das informações parciais para confirmação de nexo causal. O aprimoramento das informações do SINAN e do SIM é passo fundamental para a implantação de medidas efetivas de prevenção e controle dos acidentes de trabalho. Avaliações da cobertura, qualidade e confiabilidade das informações do SINAN e do SIM são absolutamente necessárias para que eles, de fato, cumpram o seu papel.

A cobertura do SIM pode ser considerada adequada em $\mathrm{BH}$, mas as informações sobre as circunstâncias das mortes por causas externas, obtidas nas DO ainda deixam a desejar, mesmo após a investigação no $\mathrm{IML}^{7}$. Encaminhamentos médicos, boletins de ocorrência e outras fontes consultadas mostram-se insuficientes para o esclarecimento completo dessas mortes. Se as informações dadas pelos familiares das vítimas e as constantes nos prontuários do IML fossem incorporadas às DO, elas propiciariam acesso mais fácil e ágil a dados fundamentais para o desenvolvimento de ações de vigilância em saúde ${ }^{12}$.

O importante sub-registro de óbitos por acidentes de trabalho devidos à incorporação da violência urbana, por meio de latrocínios cometidos contra trabalhadores - especialmente vigias e frentistas, já foi observado ${ }^{13}$, e torna premente a necessidade de mudanças no SIM, para que possam ser incorporados.

A falta de integração automatizada entre os sistemas de informação oficiais em saúde, como é o caso do SINAN e SIM, possibilitou que os dados de óbitos por AT permanecessem estanques em cada um deles. É necessário, também, que haja um fluxo sistemático de informações, pelo menos entre os sistemas geridos pelo MS. O Cartão SUS poderá representar o meio pelo qual o relacionamento de dados venha a ser feito sistematicamente.

\section{Colaboradores}

EF Drumond e JM Silva participaram igualmente de todas as etapas de elaboração do artigo. 


\section{Referências}

1. Santana V, Nobre L, Waldvogel BC. Acidentes de trabalho no Brasil entre 1994 e 2004: uma revisão. Cien Saude Colet 2005; 10(4):841-855.

2. Waldvogel BC. A população trabalhadora paulista e os acidentes do trabalho fatais. São Paulo Perspec 2003; 17(2):42-53.

3. Beraldo OSS, Medina MG, Borba EA, Silva LP. Mortalidade por acidentes do trabalho no Brasil: uma análise das declarações de óbito de 1979-1988. Inf Epidemiol SUS 1993; 2(1):41-54.

4. Brasil. Ministério da Saúde (MS). Portaria 777/GM, de 28 de abril de 2004. Dispõe sobre os procedimentos técnicos para a notificação compulsória de agravos à saúde do trabalhador em rede de serviços sentinela específica, no Sistema Único de Saúde SUS. Diário Oficial da União 2004; 29 abr.

5. Brasil. Ministério da Saúde (MS), Secretaria de Vigilância em Saúde, Departamento de Vigilância Epidemiológica. Sistema de Informação de Agravos de Notificação - SINAN: normas e rotinas. Brasília: MS; 2007.

6. Brasil. Ministério da Saúde. Secretaria de Vigilância em Saúde. Portaria no 116, de 11 de fevereiro de 2009. Regulamenta a coleta de dados, fluxo e periodicidade de envio das informações sobre óbitos e nascidos vivos para os Sistemas de Informações em Saúde sob gestão da Secretaria de Vigilância em Saúde. Diário Oficial da União 2009; 12 fev.

7. Cordeiro R, Binder MCP. Sub-registro de acidentes do trabalho em localidade do Estado de São Paulo, 1997. Rev Saude Publica 2003; 37(4):409-416.
8. Correa PRL, Assunção AA. A sub-registro de mortes por acidentes de trabalho: estudo de três bancos de dados. Epidemiol. Serv Saúde 2003; 12(4):203-212.

9. Cortez SAE. Acidente do trabalho: ainda uma realidade a ser desvendada [dissertação]. Ribeirão Preto: Faculdade de Medicina de Ribeirão Preto, Universidade de São Paulo; 2001.

10. Napoleão AA, Robazzi MLCC, Marziale MHP, Hayashida M. Causas de sub-registro de acidentes do trabalho entre trabalhadores de enfermagem. Rev. Latino-Am. Enfermagem 2000; 8(3):119-120.

11. Binder MC, Wludarski SL, Almeida IM. Estudo da tendência dos acidentes de trabalho registrados pela Previdência Social no período de 1995-1999, em Botucatu, São Paulo. Cad Saude Publica 2001; 17(4): 915-924.

12. Matos SG, Proietti FA, Barata RCB. Confiabilidade da informação sobre mortalidade por violência em Belo Horizonte, MG. Rev Saude Publica 2007; 41(1): 76-84.

13. Hennington EA, Cordeiro R, Moreira-Filho DJ. Trabalho, violência e morte em Campinas, São Paulo, Brasil. Cad Saude Publica 2004; 20(2):610-617.

Artigo apresentado em 02/06/2012

Aprovado em 02/10/2012

Versão final apresentada em 28/10/2012 